\title{
Infestação por Lynxacarus radovskyi em cães e gatos domésticos na cidade de Niterói $(\mathrm{RJ})$ : relato de caso
}

\section{Lynxacarus radovskyi infestation in domestic dogs and cats in Niterói city: case reported}

\author{
Ellen Jaffé, ${ }^{*}$ Simone Grillo, ${ }^{* *}$ Cleber Lincoln Andrade Costa, ${ }^{* * *}$ Carlos Eduardo Santos Vaz, ${ }^{* * *}$ \\ Luciana Casartelli Alves, ${ }^{* \star * * *}$ Nádia R. P. Almosny*****
}

\begin{abstract}
Resumo
O Lynxacarus radovskyi é um ácaro da pele do gato que se alimenta da superfície do pêlo. O potencial das manifestações clínicas é proporcional ao número de ácaros fixados no animal e a duração da infestação. Com o objetivo de pesquisar a presença de ectoparasitos em 10 felinos que apresentavam pontos avermelhados nos pêlos foi realizado o tricograma e observou-se a presença de ovos e exemplares adultos de $L$. radovskyi aderidos aos pêlos dos animais. Observou-se, ainda, que um canino, macho e adulto, que convivia com estes felinos, também estava parasitado por estes artrópodes, embora apresentando menor grau de infestação. Em cães, não existem relatos deste parasito na literatura mundial. Concluiu-se que, devido ao pequeno tamanho do parasito e as dificuldades no diagnóstico, é necessário um estudo amplo que avalie a ocorrência deste parasito e suas implicações na sanidade dos felinos.
\end{abstract}

Palavras-chave: Lynxacarus radovskyi, ácaro, dermatopatias, cães, gatos.

\begin{abstract}
The cat fur mite, Lynxacarus radovskyi, is a hair clasping surface feeder. Its potencial clinical significance is proportionate to the number of mites that attach to the animal and duration of infestation. With the objective to search for the presence of the parasites in 10 felines that showed red spots in their coats, the tricograme test was carried through and showed the presence of $L$. radovskyi individuals and eggs adhered to the animals' coats. It was also noticed that an adult male canine, which shared the same environment with the felines, was also infested by these arthropods in a lesser degree. World literature shows no trace of these ectoparasites in dogs. We concluded that due to this parasite's small size and the difficulties in the diagnosis, a broader study that evaluates the occurrence of the parasitic agent, and its implications to both feline and canine health becomes necessary.
\end{abstract}

Keywords: Lynxacarus radovskyi, mite, dermatopathologies, dogs, cats.

\section{Introdução}

O Lynxacarus radovskyi é um ácaro relacionado c0m dermatopatias de felinos e sua distribuição geográfica está relacionada com climas úmidos e tropicais (Craig et al., 1993; Pereira, 1996) como no Havaí (Merchant, 1993; Craig et al., 1993; Pereira, 1996; Wilkinson e Harvey, 1996; Scott et al., 2001; Akucewich et al., 2002; Medleau e Hnilica, 2003), Austrália (Greve e Gerrish, 1981; Craig et al., 1993; Merchant, 1993; Pereira, 1996; Wilkinson e Harvey, 1996; Scott et al., 2001; Medleau e Hnilica, 2003), Fiji (Greve e Gerrish, 1981; Craig et al., 1993; Merchant, 1993; Pereira, 1996; Wilkinson e Harvey, 1996; Medleau e Hnilica, 2003), Porto Rico (Greve e Gerrish, 1981; Craig et al., 1993; Pereira, 1996; Merchant, 1993; Medleau \& Hnilica, 2003), Flórida (Greve e Gerrish,
1981; Foley, 1991; Craig et al., 1993; Merchant, 1993; Wilkinson e Harvey, 1996; Scott et al., 2001; Akucewich et al., 2002; Medleau e Hnilica, 2003), Texas (Scott et al., 2001; Akucewich et al., 2002) e Brasil (Faccini e Coutinho, 1986; Pereira, 1996; Scott et al., 2001; Serra-Freire et al., 2002). Neste último, a sua ocorrência já foi reportada em São PauIo, Rio de Janeiro (Pereira, 1996; Serra-Freire et al., 2002) e Belém-PA (Serra-Freire et al., 2002), o que demonstra ser um problema crescente na população de gatos domésticos do nosso país (Pereira, 1996).

O ácaro pertence à subordem Astigmata (Foley, 1991) e família Listophoridae (Foley, 1991; Pereira, 1996) que parasitam pêlos de mamíferos (Pereira, 1996). É um ácaro alongado (Merchant, 1993; Pereira, 1996; Wilkinson e Harvey, 1996; Scott

\footnotetext{
* Graduanda em Medicina Veterinária - UFF. Rua Santa Rosa ,141, apto. 1002. Santa Rosa - Niterói, RJ.

** Médica-veterinária autônoma. Estrada Velha de Marica 154, casa 38, Maria Paula - Niterói, RJ.

*** Graduando em Medicina Veterinária - UFF. Rua Pedro Ernesto, 69, casa 9. Saúde - Rio de Janeiro, RJ.

**** Médico-veterinário autônomo. Av Santa Cruz, 543/104, bl 8, Realengo - Rio de Janeiro, RJ.

***** Graduanda em Medicina Veterinária - UFF. Rua Almirante Baltazar, 264 - São Cristóvão - Rio de Janeiro, RJ.

***** Prof. Dr. Depto. de Clínica e Patologia Clínica Veterinária - UFF. R. Vital Brazil filho,64 - Santa Rosa - Niterói, RJ. Email: mcvalny@vm.uff.br
} 
et al., 2001) e achatado lateralmente (Greiner, 1999), medindo 430 a $520 \mu \mathrm{m}$ de comprimento (Scott et al., 2001).

O parasito possui a porção anterior com revestimento marrom e o restante branco (Greiner, 1999) sendo incomum em pêlos de gatos (Moriello,1994; Greiner, 1999). Todas as patas possuem ventosas terminais (Merchant, 1993; Scott et al., 2001) e ele se agarra na haste do pêlo (Greve e Gerrish, 1981; Craig et al., 1993; Merchant, 1993; Moriello,1994; Pereira, 1996; Wilkinson e Harvey, 1996; Greiner, 1999; Scott et al., 2001; Medleau e Hnilica, 2003) com os palpos e os dois primeiros pares de patas (Foley, 1991; Merchant, 1993; Wilkinson e Harvey, 1996; Scott et al., 2001;) sendo rápida a sua movimentação (Craig et al., 1993).

Aparentemente, este pequeno parasito permanece por toda a sua vida aderido ao pêlo do hospedeiro (Greve e Gerrish, 1981) e supõe-se que sua alimentação seja da superfície do pêlo, o que provavelmente torna a maioria dos casos assintomáticos. Pouco se conhece sobre a biologia deste ácaro (Foley, 1991; Pereira, 1996).

A maioria dos casos de infestações é subclínica (Wilkinson e Harvey, 1996). Os sinais clínicos são potencialmente proporcionais ao número de ácaros que se fixam nos pêlos do animal e a duração da infestação (Pereira, 1996; Scott et al., 2001). Entretanto, com relação ao prurido não há associação com o grau de infestação. Pouca evidência de prurido é observada nos hospedeiros com grande número de $L$. radovskyi. $\mathrm{O}$ ato de se coçar tem sido reportado em gatos com poucos ácaros e assim mesmo pode ser indicativo de hipersensibilidade (Craig et al., 1993).

As manifestações clínicas mais comumente observadas são discreto prurido, aparência da pelagem de polvilhado de "sal e pimenta" pela visibilidade dos ácaros nos pêlos e descamação. Pode ocorrer alopecia devido ao arrancamento fácil dos pêlos, presença excessiva de caspas e dermatite miliar (Greve e Gerrish, 1981; Foley, 1991; Craig et al., 1993; Merchant, 1993; Moriello, 1994; Pereira, 1996; Wilkinson e Harvey, 1996; Scott et al., 2001; Serra-Freire et al., 2002; Medleau e Hnilica, 2003).

A superfície da pele tem uma aparência normal, mas em alguns casos pode causar erupções crostosas e exsudativas acompanhada de prurido (Greve e Gerrish, 1981).

As regiões do corpo mais acometidas pelo $L$. radovskyi são principalmente o dorso (Medleau e Hnilica, 2003) e membros posteriores (Craig et al., 1993; Greve e Gerrish, 1981). Todavia, pode ser encontrado na região cervical e torácica (Craig et al., 1993), base da cauda, região epigástrica, tendendo a se tornar uma infestação generalizada (Serra-Freire et al., 2002).

Em alguns animais a infestação pelo ácaro está associada à doença crônica e debilitante (Greve e Gerrish, 1981) ou a distúrbios gastrintestinais devido à excessiva lambedura podendo ocorrer vômito, constipação, irritação ou prolapso retais e bolas de pêlo (Foley, 1991).

A transmissão ocorre por contato direto, mas os fômites também podem ser uma importante via de transmissão (Craig et al., 1993; Scott et al., 2001). Há relatos de casos em humanos que manipulam gatos infestados aparecendo lesões na forma de erupções cutâneas papulares (Foley, 1991).

A linxacariose deve ser diferenciada da queiletielose e pediculose (Merchant, 1993; Wilkinson e Harvey, 1996) sendo que pode haver infestação concorrente com Cheyletiella spp. (Moriello, 1994). O diagnóstico definitivo é feito pelo isolamento do ácaro (Pereira, 1996; Wilkinson e Harvey, 1996) por meio de raspado de pele ou impressão com fita de acetato (Merchant, 1993; Moriello, 1994; Scott et al., 2001; Serra-Freire et al., 2002; Medleau e Hnilica, 2003).

O tratamento deve ser feito com a aplicação de duas doses de $300 \mu \mathrm{g} / \mathrm{kg}$ de ivermectina por via subcutânea com intervalo de duas semanas (Foley, 1991; Moriello, 1994; Medleau e Hnilica, 2003). Autores sugerem uma avaliação desta com maior número de casos (Pereira, 1996). Outras formas de tratamentos sugeridos incluem banhos à base de sulfeto de cálcio a 2\% (Moriello, 1994; Scott et al., 2001; Medleau e Hnilica, 2003), malation a 0,5\% (Craig et al., 1993), sulfeto de selênio, tetra-etil-tiuran (Serra-Freire et al., 2002) e banhos a base de piretrina (Craig et al., 1993; Foley, 1991; Moriello, 1994; Medleau e Hnilica, 2003) uma vez por semana durante quatro semanas (Craig et al., 1993).

O objetivo deste trabalho é relatar a ocorrência de $L$. radovskyi em gatos domésticos e também em um cão (Figura 4) na Cidade de Niterói, RJ. Esta é a primeira vez que é relatada a ocorrência de L. radovskyi em cães.

\section{Relato de caso}

Em janeiro de 2003, no bairro de Pendotiba, no município de Niterói, RJ, foi detectada a presença de ectoparasitos fixados aos pêlos de 10 gatos e um cão (Figura 4) que convivem na mesma residência. Foram coletadas amostras de pêlos de todos os animais, sendo fixadas com álcool a $70 \%$ e enviadas ao Laboratório de Patologia Clínica da Universidade Federal Fluminense, onde as amostras foram examinadas em microscopia óptica (100x e 400x).

Os animais apresentavam pouca evidência de prurido e os pêlos podiam ser arrancados facilmente. Os gatos com pêlos esbranquiçados apresentavam aspecto da pelagem de polvilhado de "sal e pimenta", havendo pontos com coloração de ferrugem (Figura 1). Durante a realização do tricograma foram observados numerosos ácaros da espécie $L$. radovskyi (Figura 2) e seus respectivos ovos (Figura 3) em todos os animais, inclusive no cão (Figura 4).

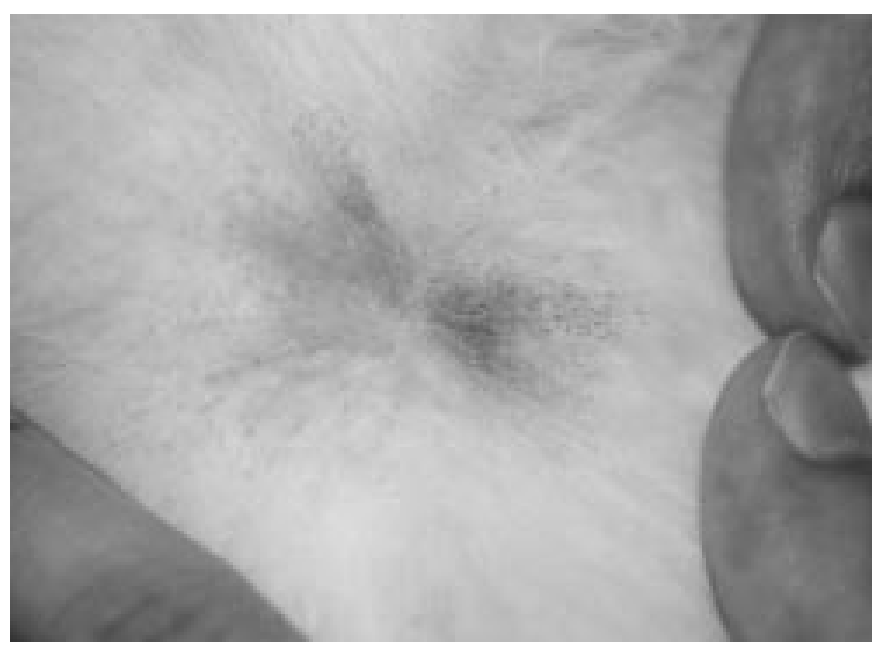

Figura 1 - Pêlos esbranquiçados de gato apresentando aspecto da pelagem de polvilhado de "sal e pimenta", havendo pontos com coloração de ferrugem 


\section{Discussão}

O parasito observado possuía a porção anterior com revestimento marrom e o restante branco com ventosas terminais nas patas, conforme relatos da literatura (Greiner, 1999).

Os animais apresentavam pouca evidência de prurido, mas os pêlos podiam ser arrancados facilmente. Os gatos com pêlos esbranquiçados apresentavam pelagem com aspecto de polvilhado de "sal e pimenta", havendo pontos com coloração de ferrugem. Estes dados estão de acordo com a literatura (Greve e Gerrish, 1981; Foley, 1991; Craig et al., 1993; Merchant, 1993; Moriello, 1994; Pereira, 1996; Wilkinson e

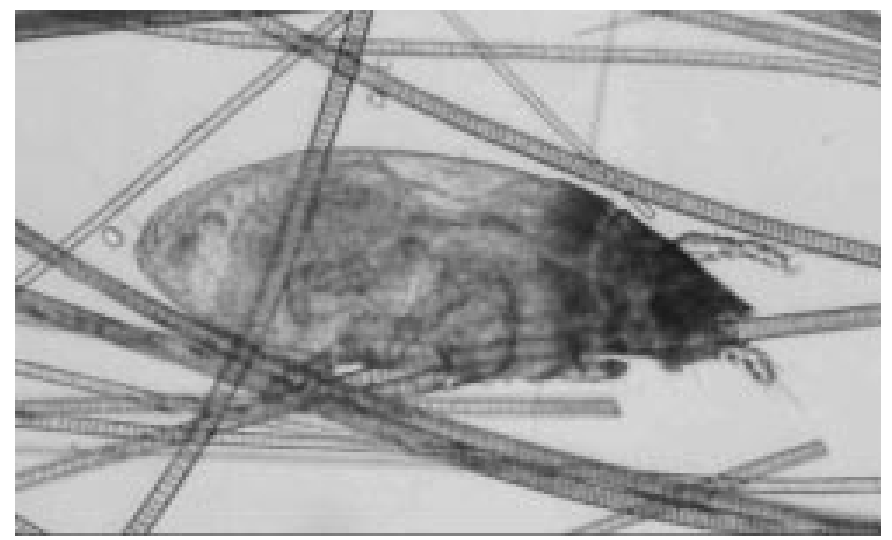

Figura 2 - L. radovskyi fêmea (aumento 400x) aderido ao pêlo de gato apresentando um ovo no seu interior

O cão apresentava-se assintomático havendo poucos ácaros na superfície do pêlo. Acredita-se que sua transmissão tenha
Harvey, 1996; Scott et al., 2001; Serra-Freire et al., 2002; Medleau e Hnilica, 2003) onde são descritos casos assintomáticos (Wilkinson \& Harvey, 1996) que podem sofrer agravamento, onde poderão ser observadas erupções crostosas e exsudativas acompanhadas de prurido, doença crônica debilitante ou distúrbios gastro-intestinais (Greve e Gerrish, 1981).

As regiões do corpo mais acometidas pelo L. radovskyi eram principalmente o dorso (Medleau e Hnilica, 2003) e membros posteriores (Greve e Gerrish, 1981; Craig et al., 1993) mas, de uma forma geral, todo o corpo dos animais estavam afetados.

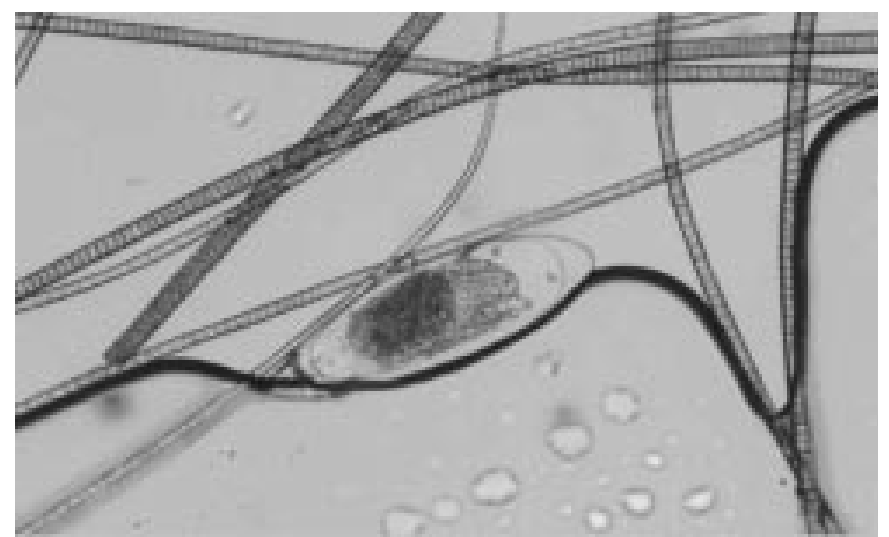

Figura 3 - Presença de ovo de L.radovskyi (aumento de 400x) aderido ao pêlo de gato

ocorrido devido a infestação maciça na qual os gatos estavam acometidos e que tenha ocorrido por contato direto.

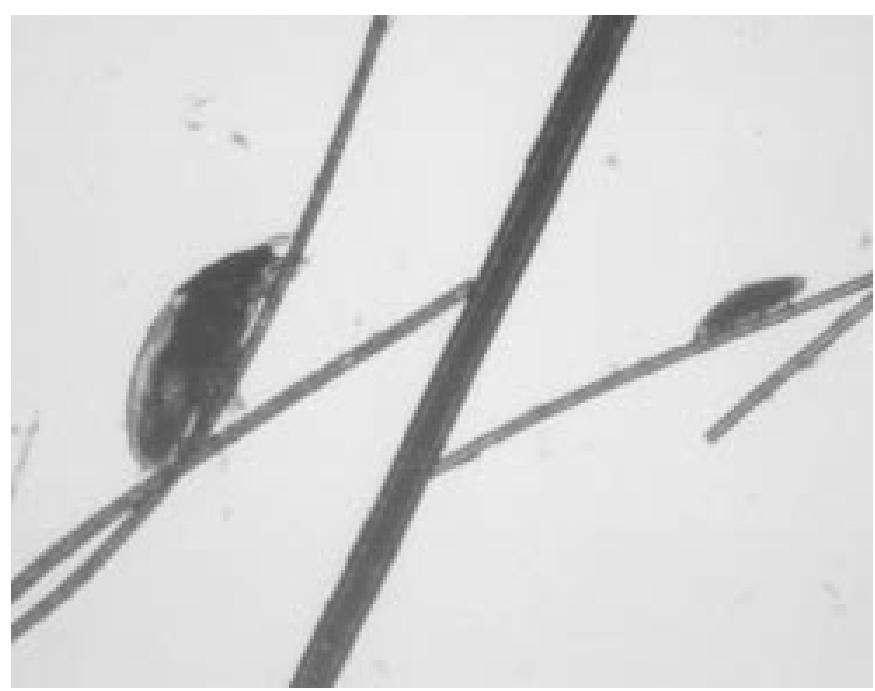

Figura 4 -- L. radovskyi sendo um exemplar adulto (esquerda) e um ovo (direita) (aumento 100x) aderido aos pêlos de cão

\section{Conclusões}

Muitos dos animais parasitados podem apresentar-se assintomáticos, entretanto, é necessário um estudo mais amplo que avalie suas conseqüências na clínica de caninos e felinos. O pequeno tamanho do parasito dificulta o diag- nóstico clínico, sendo necessário um tricograma, assim como estudo amplo que avalie a ocorrência deste parasito e suas implicações na sanidade dos felinos e caninos. Contudo, no cão é importante que se realizem maiores estudos relacionados com o ácaro especificando, suas conseqüências, uma vez que não há pesquisas sobre o referido assunto. 


\section{Referências}

AKUCEWICH, L. H.; PHILMAN, K.; CLARK, A.;GILLESPIE, J.;KUNKLE, G.; NICKLIN, C. F.; GREINER, E. C. Prevalence of ectoparasites in a population of feral cats from north central Florida during the summer. Veterinary Parasitology. v. 109, 2002, p. 129-139.

CRAIG, T. M.; TELL, P. D.; DUBUISSON, L. M.; DUBUISSON R. K. Lynxacarus radovskyi infestation in a cat. Journal of American Veterinary Medical Association. v. 202, n. 4, 1993, p. 613-614.

FACCINI, J. L. H., COUTINHO, V. Ocorrência de Lynxacarus radovskyi (Acari: Listophoridae) em gato doméstico no Brasil. Arquivos da Universidade Federal Rural do Rio de Janeiro, v. 9, n. 1-2, p. 91-93, 1986.

FOLEY, R. H. An Epizootic of a rare fur mite in an island's cat population. Feline Practice. v. 19, n. 3 p. 17-119. 1991.

GREINER, E.C. Artrópodes de importância veterinária na América do Norte. In: SLOSS, M. W.; ZAJAC, A. M.; KEMP,R. L. Parasitologia clínica veterinária. 6. ed. São Paulo: Manole, 1999.

GREVE, J. H.; GERRISH, R. R. Fur mites (Lynxacarus) from cats in Florida. Feline Practice. v. 11, n. 6, p. 28-30, 1981.

MEDLEAU, L; HNILICA, K. A. Ácaro de pêlos de gatos (Lynxacarus radovskyı). In: MEDLEAU, L.; HNILICA, K. A. Dermatologia de peque- nos animais: Atlas colorido e guia terapêutico. São Paulo: Roca, 2003.

MERCHANT, S. R. The Skin: Parasitic Diseases. In: NORSWORTHY, G. D. Feline Practice.. Philadelphia: J.B. Lippincott Company, 1993, p. 515-516.

MORIELLO, K. A. Diseases of the skin. In: SHERDING, R. G. The cat:diseases and clinical management. 2. ed. New York: Churchill Livingstone, 1994, v. 2, p. 1915-1916.

PEREIRA, M. C .P. The cat fur mite (Lynxacarus radovskyi) in Brazil. Feline Practice. v. 24, n. 5, p. 24-26, 1996.

SERRA-FREIRE, N. M.;BENIGNO, R. N. M.; OLIVEIRA, S. A.; GALVÃO, G. Lynxacarus radovskyi- diagnóstico e tratamento em felinos de BelémPará. Revista Universidade Rural, Série. Ciências de Vida, v. 22, n. 1, p. 57-60, 2002.

SCOTT, D. A.; MILLER, W. H.; JR.; GRIFFIN, C. E. Parasitic Skin Disease. In: SCOTT, D. A.; MILLER, W. H.; JR.; GRIFFIN, C. E. Muller \& Kirk's small animal dermatology. 6. ed. Philadelphia:W.B.Saunders Company, 2001, p. 446-447.

WILKINSON, G. T.; HARVEY, R. G. Doenças Parasitárias. In:WILKINSON, G. T.; HARVEY, R.G. Atlas colorido de dermatologia dos pequenos animais: guia para o diagnóstico. 2. ed. São Paulo: Manole, 1996. 